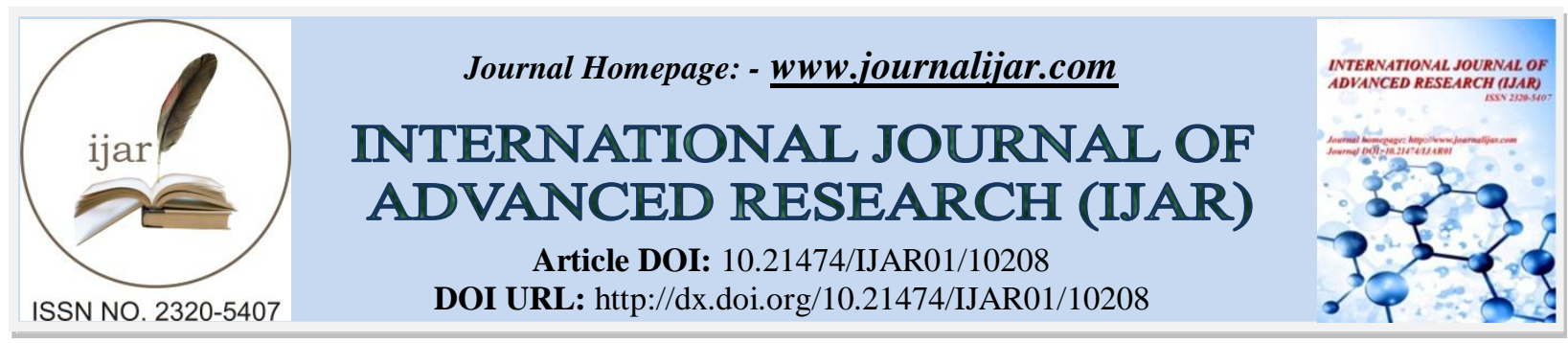

RESEARCH ARTICLE

\title{
GROSSESSE EXTRA - UTERINE AU CENTRE HOSPITALIER NATIONALE DE NOUAKCHOTT : ASPECTS EPIDEMIOLOGIQUES, CLINIQUES ET THERAPEUTIQUES
}

\author{
Fatimetou Abdelkader
}

\section{Manuscript Info}

\section{Manuscript History}

Received: 10 October 2019

Final Accepted: 12 November 2019

Published: December 2019

Key words:-

Grossesse Extra, Uterine,
Epidemiologie, Clinique, Therapeutique

\begin{abstract}
La grossesse extra-utérine (GEU) constitue une pathologie grave en prémier trimestre de grossesse. Elle est la prémiere cause de la mortalite maternelle en prémier trimestre. Son diagnostic et la prise en charge précoce éviterait les complications. Notre objectif était d'évaluer les grossesses extra-utérines à la maternité du centre hospitalier nationale de Nouakchott. Nous avons mené une étude transversale descriptive avec recrutement prospectif des données, du premier mai 2013 au 30 avril 2014 au service de gynécologieobstétrique. Les informations ont été recueillies à l'aide d'un questionnaire anonymée préétablie. Un total de 44 cas de GEU pour 4409 accouchements ont été enregistrés, soit une fréquence globale de $0,99 \%$. La prise en charge était essentiellement chirurgicale par laparotomie, radicale dans 93,2\%. Le taux de survie était de $100 \%$. La GEU reste une pathologie fréquente dans notre milieu, avec une fréquence hospitalière de $0,99 \%$ des naissances vivantes. La prise en charge est essentiellement chirurgicale par laparotomie. Aucun cas de deces n'a été retrouvé.
\end{abstract}

Copy Right, IJAR, 2019,. All rights reserved.

\section{Introduction:-}

La grossesse extra-utérine (GEU) correspond à la nidation et au développement de l'œuf en dehors de la cavité utérine $[1 ; 2]$. C'est une pathologie grave, un exemple de l'extrême urgence chirurgicale au premier trimestre de la grossesse. Elle présente la première cause de mortalité du premier trimestre de la grossesse et compromet significativement la fertilité ultérieure [3].Son incidence varie entre 1 à $2 \%$ des grossesses [1; 4]. L'incidence de la GEU dans les pays développés est de l'ordre de 100 à 175 par an pour 100000 femmes âgées de 15 à 44 ans [3]. Plusieurs études récentes ont révélé l'augmentation constante de la fréquence de la GEU, une augmentation liée à l'expansion des infections sexuellement transmissibles (IST) et du tabac qui sont associés au risque important de séquelles et sont un marqueur de santé publique justifiant un diagnostic précoce [5]. Ces dernières années, beaucoup de progrès ont été réalisés en vue d'améliorer la prise en charge et le pronostic de la GEU essentiellement dans les pays développés : apport de l'échographie pelvienne (endovaginale) et des dosages biologiques sanguins (bêta hCG plasmatique, progestérone). Le traitement médical, la cœlioscopie diagnostique et opératoire conservatrice permettent, dans les formes précoces, d'améliorer la prise en charge globale et la fertilité ultérieure.

\section{Methodologie:-}

C' est une étude transversale descriptive avec recrutement prospectif des données portant sur les patientes hospitalisées et traitées pour GEU au service de maternité du Centre Hospitalier National durant une période d'un 
an, c'est-à-dire au cours de la période allant du premier mai 2013 au 30 avril 2014. L'étude a recensé de façon exhaustive tous les cas de GEU dans le service pendant la période de l'étude. Une fiche d'enquête a été établie pour le recueil des données, La saisie et l'analyse des données ont été effectuées sur le logiciel SPSS, version 20, Microsoft Office Excel 2007. Les résultats sont exprimés en nombre, moyenne, extrêmes, et en pourcentage (\%).

\section{Resultats:-}

Un total de 44 cas de GEU pour 4409 accouchements ont été enregistrés, soit une fréquence globale de $0,99 \%$. Deux pics de fréquence en mai 2013 et février 2014 de 8 patientes par mois soit une incidence respectivement de 2,6\% ; $2,9 \%$

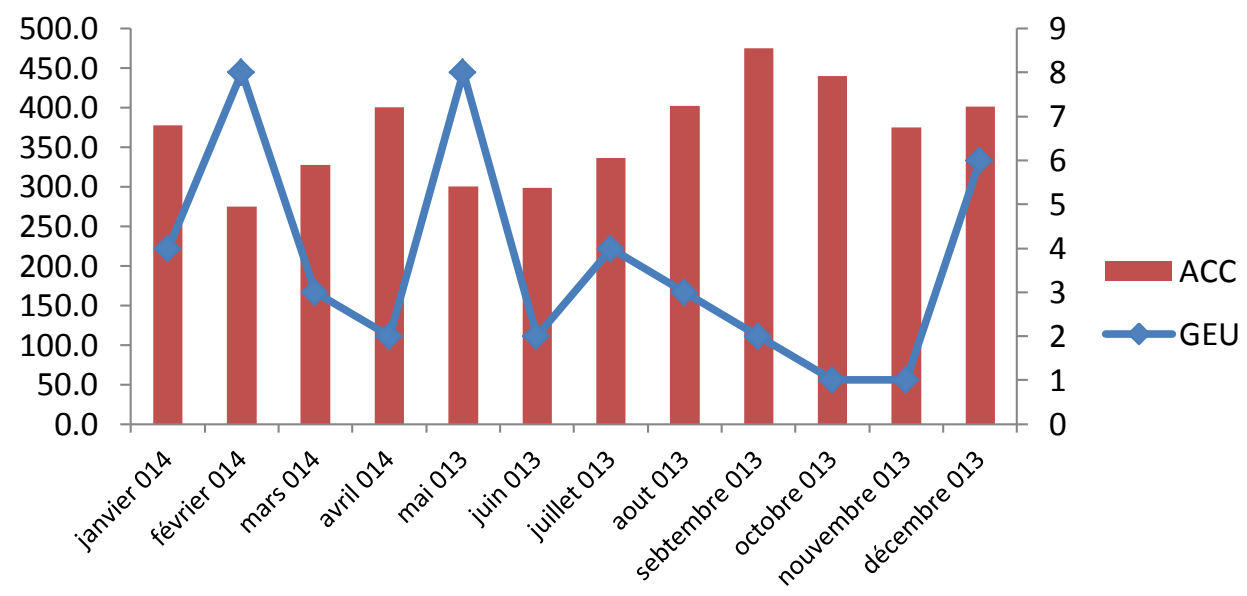

Figure 1:- Nombre des accouchements et GEU par mois

Près d'un quart de nos patientes étaient évacué de l'intérieur du pays (22,73\%) et plus de la moitièe entr eux avait un niveau socio-économique bas $(68,2 \%)$. La race noire a été la plus touché dans $68,2 \%$ de cas. L'âge moyen de nos patientes était de 29,84 $\pm 5,988$ ans avec des extrêmes allant de 15 à 44 ans, la tranche d'âge la plus touchée 25 à 34 était de $61,4 \%$. Les paucipares étaient les plus touchées avec un taux de 54,6\% et les multigestes represente $82 \%$ des cas.

Les ATCD de chirurgie abdomino-pelvienne étaient de 13,6\%. Les antécédents d'IST ont été les plus fréquents $(38,6 \%)$ suivis de près par les contraceptions orales $(36,3 \%)$ puis les avortements $(25 \%)$ et l'infertilité $(18 \%)$

Tableau 1:- ATCD gynéco-obstétricaux.

\begin{tabular}{|l|l|l|}
\hline Antécédents & Nombre de cas & Fréquence $(\%)$ \\
\hline IST & 17 & 36,8 \\
\hline Contraception orale & 16 & 36,3 \\
\hline Avortements spontanés & 11 & 25 \\
\hline Stérilité I & 4 & 9,0 \\
\hline Stérilité II & 4 & 9,0 \\
\hline Grossesse induite & 2 & 4,5 \\
\hline Contraception injectable & 1 & 2,3 \\
\hline
\end{tabular}

Le principal motif de consultation était la douleur pelvienne chez $91 \%$ de nos patientes suivi de l'aménorrhée $(86,3 \%)$ et des métrorragies $(61,3 \%)$. Ces symptômes étaient associés chez $(40,9 \%)$ des patientes. 10 de nos patientes ont presté une lipothymie et/ou malaise avant son admission,

Tableau 2:- Signes Fonctionnels.

\begin{tabular}{|l|l|l|}
\hline Signes fonctionnels & Nombres de cas & Pourcentage \% \\
\hline Douleur pelvienne & 41 & 91 \\
\hline Aménorrhée & 38 & 86,3 \\
\hline Métrorragie & 27 & 61,4 \\
\hline
\end{tabular}




\begin{tabular}{|l|l|l|}
\hline Lipothymie et/ou malaise & 10 & 22,7 \\
\hline Signes sympathiques de grossesse & 7 & 15,9 \\
\hline La triade classique de GEU & 18 & 40,9 \\
\hline
\end{tabular}

Quinze patientes étaient admises en état de choc, soit $34 \%$ dans notre série, en présence de chiffre tensionnel bas et un pouls filant.

Le traitement chirurgical était radical dans $93,2 \%$ avec une salpingectomie dans $86,4 \%$, une annexectomie dans $6,8 \%$ des cas et conservateur chez 3 patientes (6.8\%), avec salpingotomie dans 2 cas et un cas d'expression tubaire La localisation tubaire de GEU était la plus fréquente dans 38 cas soit, $86,2 \%$, la grossesse hétérotopique était objectivée dans 3 cas soit, $6,8 \%$ et la grossesse abdominale dans un seul cas soit, $2,3 \%$.

Tableau 3:- Situation anatomique de GEU.

\begin{tabular}{|l|l|l|l|}
\hline Situation & Nombres de cas & Pourcentage(\%) \\
\hline Tubaire & Ampullaire & 22 & 50 \\
\hline & Isthmique & 4 & 9,0 \\
\hline & Interstitielle & 3 & 6,8 \\
\hline & Pavillonnaire & 2 & 4,6 \\
\hline & en cours d'avortement & 3 & 6,8 \\
\hline & Sans précision & 4 & 9,0 \\
\hline Hétérotopique & 3 & 6,8 \\
\hline Ovarienne & 1 & 2,3 \\
\hline Abdominal & 1 & 2,3 \\
\hline Corne rudimentaire & 1 & 2,3 \\
\hline Total & 44 & 100 \\
\hline
\end{tabular}

\section{Discussion:-}

La GEU occupe une place privilégiée dans la littérature médicale et demeure une pathologie fréquente dans le monde. Après une forte augmentation entre 1970 et 1990, l'incidence de la GEU a décru globalement pendant une dizaine d'années. Actuellement la tendance n'est pas univoque puisque l'incidence augmente chez les femmes sans contraception alors qu'elle diminue chez les autres [6]. L'incidence de la GEU varie entre 1 et $2 \%$ des grossesses Cette incidence a doublé voire triplé au cours des deux dernières décennies dans les pays industrialisés [1; 7]. Dans notre série, cette incidence est de l'ordre de $0,99 \%$, un résultat proche de celui du Rafia [8] (0.92\%) et du Lofredo [9] $(0,84 \%)$. Notre taux est cependant inférieur à celui des séries africaines : Dembele [10] (1,5\%), Sy T [11] $(1,4 \%)$ et Randia [1] (2,48\%). Des études américaines signalent que les femmes noires étaient plus susceptibles d'avoir une grossesse extra-utérine par rapport aux blanches [12]. Dans notre série, nous avons trouvé 68,2\% de cas chez les patientes noires. L'âge semble être associé à un risque accru de GEU, indépendamment du fait de l'allongement de la période d'exposition aux différents facteurs de risque [3]. Il en découle que la fréquence de la GEU diminue avec les âges extrêmes pour augmenter durant la période active de la vie sexuelle. Dans notre travail la tranche d'âge 25-34 ans a été la plus concernée (61,4\%), avec un âge moyen de 29,84 ans, nos résultats sont similaires à ceux trouvés par Jessica et al [7] aux Etats Unis où la tranche d'âge la plus rependue était 25-35 ans alors que dans l'étude effectuée par Methlouthi, [13] les patientes ayant moins de 30 ans ont été les plus concernées soit 58\% avec un âge moyen 25,4 ans. Dans la plupart des études, la GEU est classiquement associée à une faible parité $[8 ; 14 ; 15]$. Ceci concorde avec les données de notre série ou les paucipares étaient les plus touchées $(54,6 \%)$ et les nullipares représentent $18,2 \%$ de cas. La GEU est une pathologie multifactorielle, selon la littérature, les principaux facteurs de risque de GEU chez les femmes sans contraception sont les antécédents d'infection génitale, de chirurgie tubaire et le tabac [1]. Le rôle directement causal de l'infection génitale est très vraisemblable ainsi Les IST et leurs complications (salpingites et pelvipéritonites...) représentent le facteur de risque principal de GEU. Le germe le plus fréquemment en cause semble être le Chlamydia trachomatis. Les infections génitales expliqueraient $50 \%$ des cas de GEU par l'intermédiaire d'un mécanisme d'altération séquellaire de l'anatomie tubaire. La fréquence des formes frustres ou infra cliniques de salpingites explique que la sérologie à Chlamydia soit le témoin principal de l'antécédent d'infection pelvienne, avec une multiplication du risque entre 3 et 8 ainsi observé en Suède une diminution de l'incidence de GEU après des actions de prévention qui avaient fait diminuer le taux d'infections génitales à chlamydia $[3 ; 7 ; 12]$. Les femmes ayant des rapports protégés par préservatifs ont un risque de GEU plus faible que les non-utilisatrices $[6 ; 14 ; 12]$. Dans notre étude, le taux des IST représentait 38,6\%. Ce taux élevé de maladies sexuellement transmissibles pourrait être lié aux habitudes socioculturelles et à la diversité 
ethnique dans un pays arabo africain comme le nôtre où la polygamie et le divorce sont à l'origine de la multiplicité des partenaires $(54,5 \%)$ ce qui favorise sans doute les IST.

L'association de triade (algie pelvienne, retard des règles et métrorragies) a été trouvée chez $40,9 \%$ de nos patientes, des résultats inférieurs à ceux trouvés par Randria D.M.A [1] 78\% et Rafia [8] 44,18\%.

Dans notre série la localisation tubaire a représenté $86,5 \%$, dont plus de la moitié (58\%) étaient ampullaires et $(10,5 \%)$ isthmiques, des résultats conformes à ceux de la littérature africaine: Pour Sy T [11] la localisation tubaire était de $(86,3 \%)$ avec une prédominance ampullaire de $(43,1 \%)$, pour Nayama et [5] ces taux étaient de $88.11 \%$.

Le traitement a été chirurgical par laparotomie pour l'ensemble de nos patientes. Le taux élevé de traitement radical était lié à la consultation tardive de nos patientes au stade de complications par rupture de GEU.

Dans une étude américaine récente sur une durée de 9 ans le recours à la laparotomie classique était de $70.6 \%$ des cas [7].

\section{Conclusion:-}

La GEU est une pathologie grave qui présente une urgence vitale et fonctionnelle. Elle est fréquente partout dans le monde et touche surtout la femme jeune. En Mauritanie, la GEU constitue un problème de santé publique. Elle concerne surtout les femmes jeunes (25-34 ans) et 54,6\% de nos patientes étaient paucipares, la majorité étant multigestes. L'antécédent de IST (36,8\%), est le principal facteur de risque retrouvé. Le statut hémodynamique des patientes est stable dans la majorité des cas. La prise en charge, essentiellement chirurgicale, est radicale dans 93,2\% des cas. Le taux de survie est de $100 \%$ dans notre série.

\section{References:-}

1. Randriambololona D.M.A., Randiambololona N.T. et al. Grossesse extra-utérine à Madagascar. Médecine et Santé Tropicales. 2012;22:394-397.

2. Cabar FR, Fettback PB, Zugaub M. Serum markers in the diagnosis of tubal pregnancy. Clinics (Sao Paulo). 2008;63:701-8.

3. Gervaise A, Fernandez H. Prise en charge diagnostique et thérapeutique des grossesses extra-utérines. J Gynecol Obstet Biol Reprod. 2010;39:F17-24.

4. Sivalingam VN, Duncan WC, Shephard LA, Horne AW. Diagnosis and management of ectopic pregnancy. J Fam Plann Reprod Health Care. 2011;37:231-40.

5. Nayama M,Gallais A,Ousmane N, et al. Prise en charge de la grossesse extra-utérine dans les pays en voie de développement : exemple d'une maternité de référence au Niger. Gynecol Obstet Fertil. 2006;34:14-8.

6. Bouyer.J. Epidemiolgie de la grossesse extra-utérine: Incidence, facteurs de risque et conséquences. J.Gynecol.Obstet.Biol.Reprod. 2003, 32(7) :8-17.

7. Jessica Papillon-Smith et Al. Population-Based Study on the Effect of Socioeconomic Factors and Ra ce on Management and Outcomes of 35,535 Inpatient Ectopic Pregnancies. Journal of Minimally Invasive Gynecology. September/October 2014;21(5):914-920.

8. Rafia Mohamed. Prise en charge de la grossesse extra-utérine au service de gynéco-obstétrique "B" (A propos de 86 cas). Thèse .Med. Casa 2006,137.

9. Loffredo.V, Tesquier.L, Paris F.X, Debrux .J. La grossesse extra-utérine. ncycl. Méd. Chir. 1984; 700- A10, 20p.

10. Yaya Dembele. Grossesse extra-utérine : Aspects épidémiologique, clinique, diagnostique, thérapeutique et pronostique au centre de santé de référence de la commune $\mathrm{V}$ du distrit de Bamako a propos de 128 cas. Thèse. Med. Bamako. 2006.

11. Sy T, Diallo Y et al. Prise en charge de la grossesse extra-utérine à Conakry (Guinée). Med Trop. $2009 ; 69$ :565-568.

12. Debra B. Stulberg, M.D., M.A. Ectopic pregnancy rates and racial disparities in the Medicaid population, 20042008. Fertility and Sterility. Déc $2014 ; 102(6): 1671-1676$.

13. N. Mathlouthi, O. Slimani, A. Fatnassi, R. Ben Temime, et al. Diagnostic échographique des grossesses extrautérines : Etude prospective à propos de 200 cas. La tunisie Medicale. 2013;91(04):254-257.

14. Jean Bouyer, PH.D. Emmanuelle Rachou, et al. Risk factors for extrauterine pregnancy in women using an intrauterine device. FERTILITY AND STERILITY. NOVEMBER 2000;74(5).

15. Lawani et al. Ectopic pregnancy: a life-threatening gynecological emergency. Int J Womens Health. 2013;5:515-521. 